\title{
How active are children in Toronto? A comparison with accelerometry data from the Canadian Health Measures Survey
}

\author{
M. R. Stone, PhD (1); G. E. Faulkner, PhD (2); R. N. Buliung, PhD (3)
}

This article has been peer reviewed.

\begin{abstract}
Introduction: The Canadian Health Measures Survey (CHMS) is the most comprehensive direct health measures survey ever conducted in Canada. Results show that the majority of children and youth ( $93 \%$ ) do not meet current physical activity recommendations for health. CHMS data have not yet been considered alongside an independent sample of Canadian youth; such a Canadian-context examination could support CHMS results and contribute to discussions regarding accelerometry data reduction protocols.
\end{abstract}

Methods: From 2010 to 2011, valid accelerometry data were collected on 856 children living in the Greater Toronto Area (GTA). Where possible, data presentation and analyses were aligned with the CHMS protocol such that physical activity outcomes could be compared.

Results: Overall, trends were similar, with some deviations likely due to contextual and sampling differences and differences in data collection/reduction protocols regarding accelerometer model selection, wear time, activity intensity thresholds and epoch.

Conclusion: The similar trends support the notion that physical inactivity is an ongoing problem in communities across Canada.

Keywords: ActiGraph, accelerometer, physical activity, sedentary behaviour, obesity, public health, youth, CHMS

\section{Introduction}

Regular physical activity in childhood is associated with many physical, physiological and mental health benefits. ${ }^{1}$ Canada's physical activity guidelines suggest children and adolescents aged 5 to 17 years accumulate at least 60 minutes of moderate-to-vigorous physical activity (MVPA) each day. ${ }^{2}$ There is also evidence that they should engage in vigorous physical activity (VPA) at least 3 days a week. ${ }^{2}$ While self-report and pedometer data have provided some evidence of national physical activity trends over time, ${ }^{3}$ direct, objective assessments using accelerometry (on a national scale) have been absent until recently.

March 2011 saw the release of physical activity and sedentary behaviour data collected on a nationally representative sample of Canadian children and youth $(n=1608$; boys $=809$; girls $=799)$ as part of the Canadian Health Measures Survey (CHMS). ${ }^{4}$ Actical accelerometers (Phillips - Respironics, Oregon, US) were used to capture minute-by-minute data over 7 consecutive days. Information was extracted using quality control and data reduction decisions ${ }^{5}$ on the amount of time children and youth typically spend sedentary and in light, moderate and vigorous intensity physical activity; the amount of time spent in MVPA; the average number of steps taken per day; and the percentage of children attaining selected physical activity criteria. Results indicated that very few (7\%) achieve recommended levels of physical activity (with more boys achieving guidelines than girls), and many spend a significant portion of their day sedentary (average of 8.6 hours per day). ${ }^{4}$

The CHMS is the most comprehensive direct health measures survey conducted in Canada. In addition to national estimates of physical activity levels, the study has also shed light on the declining levels of fitness observed in Canadian youth over the past few decades. ${ }^{6}$ These data have received considerable public interest and media attention. They have also fuelled national campaigns (i.e. ParticipACTION; www.participaction.com) to increase population-wide levels of physical activity in children and youth. Comparisons with nationally representative data from the United States ${ }^{7}$ revealed similar trends in physical activity and sedentary behaviour, despite some contextual, sampling and methodological differences between the two datasets. However, to our knowledge CHMS data have yet to be compared with an independent sample of Canadian children and youth. It would be relevant to verify their accuracy, given the widespread dissemination of the CHMS findings and their impact on research, policy and practice across Canada.

The aim of this study is to present accelerometer data from another study,

Author references:

1. School of Health and Human Performance, Dalhousie University, Halifax, Nova Scotia, Canada

2. Faculty of Kinesiology and Physical Education, University of Toronto, Toronto, Ontario, Canada

3. Department of Geography, University of Toronto Mississauga, Mississauga, Ontario, Canada

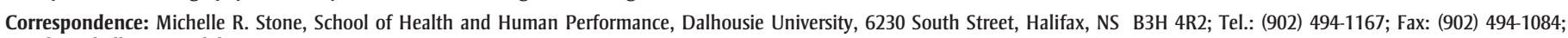
Email: michelle.stone@dal.ca 
Project BEAT, in a similar fashion to the way CHMS data have been reported, and discuss similarities and discrepancies between the two datasets.

\section{Methods}

\section{Data source}

Project BEAT (Built Environment and Active Transport; www.beat.utoronto.ca) is a large-scale, multidisciplinary and mixed method study examining how the built environment influences the way elementary school children travel to school in Toronto, Ontario. In January 2010 all elementary schools with Grade 5 and 6 students within the Toronto District School Board $(n=469)$ received an invitation to participate. Out of the pool of interested schools (54 responded, 40 of which were interested; response rate $=11.5 \%)$, 16 were selected because they varied with respect to built form (suburban looping street layout versus urban grid-based street layout) and socio-economic status (SES; low- and high-income households based on median household income reported in the 2006 Canada Census). Half of the surveyed schools were low-SES schools, and the other half were high-SES schools. Consent was obtained from participating school boards, individual schools, parents and students. Student participation was voluntary. Ethics approval from the Toronto District School Board and University of Toronto Ethics Committee was received.

\section{Participants}

Of the 1704 students enrolled in Grades 5 through 6 at the 16 participating schools, 1027 (60.3\%; boys, $n=478$; girls, $n=549$ ) completed the travel behaviour survey and were given consent to participate in the study by their parents/guardians; missing responses resulted from parent or student refusal. Prior to any data collection, children completed an assent form ( $\mathrm{n}=1001 ; 26$ students were absent for data collection). Height and weight were measured to calculate body mass index (BMI), and accelerometer-measured physical activity data were collected. For inclusion in data analysis, each child was required to wear an accelerometer for a minimum of 10 hours for at least 3 weekdays and 1 weekend day. A string of 30 minutes of consecutive zeros was classified as nonwear time/sleep time; these periods (most of which occurred during sleep) were removed from the analyses. Biologically implausible data were assessed to determine whether to include files in the final analyses. Of the 1001 children who wore an accelerometer, $95.8 \%$ had at least 1 valid day of data and $85.5 \%$ had at least 3 weekdays and 1 weekend day of valid data ( $\mathrm{n}=856$; boys $=389$, girls $=467$; Table 1 ). This article is therefore based on these 856 participants (mean age [standard deviation] 11.1 [0.6] years), who met these inclusion parameters. This final response rate $(856 / 1704=50.2 \%)$ is consistent with other active-consent studies with Canadian elementary school students. ${ }^{8}$ Using age- and sex-specific BMI cut-points provided by the International Obesity Task Force, ${ }^{9}$ participants were classified as normal weight, overweight or obese (Table 2).

\section{Measurement of physical activity and sedentary behaviour}

Children's physical activity was measured for seven days using an accelerometer (ActiGraph GT1M; Pensacola, FL, United States). The ActiGraph series are the most commonly used devices in the field, and they have moderate to good validation in children. ${ }^{10}$ Prior to data collection, the intra-unit and inter-unit variability of all ActiGraph monitors $(\mathrm{n}=120)$ was tested using a standardized treadmill protocol. The coefficients of variation were within acceptable limits. ${ }^{11,12}$

We used a 5-second epoch to capture rapid transitions in activity typical in children and related to health outcomes. ${ }^{13}$ Children were asked to wear their accelerometer consistently; they were asked to only remove the device when engaging in water-based activities. The monitors were initialized to start collecting data at 12:00 A.M. on the day they were handed out to participants. The first day was excluded from data analyses to

TABLE 1

Distribution of Project BEAT and CHMS participants, by valid days of accelerometer wear (10 or more wear hours), age group and sex

\begin{tabular}{|c|c|c|c|c|c|c|c|c|c|c|}
\hline \multirow[b]{2}{*}{ Study, Age group } & \multicolumn{10}{|c|}{ Number of valid days of accelerometer wear, $\%$} \\
\hline & $\mathbf{0}^{\mathbf{a}}$ & 1 & 2 & 3 & 4 & 5 & 6 & 7 & $\geq 1$ & $\geq 4^{b}$ \\
\hline \multicolumn{11}{|l|}{ Project BEAT } \\
\hline \multicolumn{11}{|l|}{ 10-12 years } \\
\hline Total & 4.2 & 1.8 & 1.6 & 6.9 & 2.1 & 7.9 & 21.9 & 53.6 & 95.8 & 85.5 \\
\hline Boys & 4.1 & 2.6 & 1.7 & 7.6 & 1.7 & 8.4 & 20.5 & 53.3 & 95.9 & 84.0 \\
\hline Girls & 4.3 & 1.1 & 1.5 & 6.3 & 2.4 & 7.4 & 23.0 & 53.9 & 95.7 & 86.8 \\
\hline \multicolumn{11}{|l|}{ CHMS } \\
\hline Total $^{c}$ & 4.6 & 2.9 & 3.6 & 4.1 & 8.2 & 12.7 & 24.0 & 39.8 & 95.4 & 84.7 \\
\hline \multicolumn{11}{|l|}{$6-10$ years } \\
\hline Boys & 2.7 & 2.4 & 3.2 & 1.5 & 6.4 & 11.5 & 24.7 & 47.7 & 97.3 & 90.2 \\
\hline Girls & 4.2 & 2.4 & 2.1 & 1.8 & 6.6 & 13.4 & 22.1 & 47.4 & 95.8 & 89.5 \\
\hline \multicolumn{11}{|l|}{$11-14$ years } \\
\hline Boys & 4.4 & 2.0 & 1.7 & 5.1 & 6.4 & 11.9 & 30.5 & 38.0 & 95.6 & 86.8 \\
\hline Girls & 3.2 & 2.8 & 3.6 & 2.1 & 7.8 & 12.1 & 23.1 & 45.2 & 96.8 & 88.3 \\
\hline
\end{tabular}

Sources: Built Environment and Active Transport (BEAT) Project (2010-2011); 2007-2009 Canadian Health Measures Survey $(\mathrm{CHMS})^{4}$

Abbreviations: BEAT, Built Environment and Active Transport; CHMS, Canadian Health Measures Survey.

a Agreed to wear accelerometer, but returned device with no valid data (invalid wear or device malfunctioned).

b Three weekdays and one weekend day.

c Total includes additional age group (aged 15-19 years) sampled in CHMS. Remainder of table reflects results for those aged 6-10 years and 11-14 years, in light of Project BEAT's sample demographics (aged 10-12 years). 
TABLE 2

Descriptive characteristics of Project BEAT and CHMS participants, by age group and sex

\begin{tabular}{lcccccc} 
& \multicolumn{2}{c}{ Project BEAT } & \multicolumn{2}{c}{ Canadian Health Measures Survey (CHMS) } \\
& \multicolumn{2}{c}{$\mathbf{1 0 - 1 2}$ years } & \multicolumn{2}{c}{$\mathbf{6}-\mathbf{1 0}$ years } & \multicolumn{2}{c}{ 11-14 years } \\
Characteristics & Boys & Girls & Boys & Girls & Boys & Girls \\
\hline Sample size, $\mathrm{n}$ & 389 & 467 & 369 & 340 & 256 & 248 \\
Mean age, years & 11.0 & 11.1 & 8.2 & 8.1 & 12.5 & 12.3 \\
Mean height, cm & 147.2 & 147.5 & 133.9 & 131.6 & 158.9 & 156.9 \\
Mean weight, kg & $42.3^{\mathrm{a}}$ & 40.9 & 32.5 & 29.9 & 52.1 & 50.6 \\
Mean BMI, kg/m & $19.3^{\mathrm{a}}$ & 18.6 & 17.8 & 17.0 & 20.3 & 20.4 \\
BMI category, \% & & & & & & \\
\multicolumn{1}{c}{ Normal } & $67.4^{\mathrm{a}}$ & 73.9 & 74.4 & 82.5 & 72.5 & 70.5 \\
Overweight & 21.9 & 21.6 & $17.1^{\mathrm{E}}$ & $12.6^{\mathrm{E}}$ & 21.5 & $23.0^{\mathrm{E}}$ \\
Obese & $10.8^{\mathrm{a}}$ & 4.5 & $8.1^{\mathrm{E}}$ & $4.9^{\mathrm{E}}$ & $6.0^{\mathrm{E}}$ & $6.5^{\mathrm{E}}$ \\
\hline
\end{tabular}

Sources: Built Environment and Active Transport (BEAT) Project (2010-2011); 2007-2009 Canadian Health Measures Survey (CHMS). ${ }^{4}$

Abbreviations: BEAT, Built Environment and Active Transport; BMI, body mass index; CHMS, Canadian Health Measures Survey.

${ }^{a}$ Significantly different from girls; $p<.05$.

b International Obesity Task Force classification. ${ }^{9}$

${ }^{\mathrm{E}}$ Use with caution.

control for any participant reactivity and because they were often handed out midday. Data collection took place during the Spring/Summer (April to June) and Fall (September to December) school period to limit any seasonal effect.

Time spent at various levels of movement intensity (sedentary, light, moderate, vigorous, hard) was classified according to published thresholds in children ${ }^{14}$ and used to determine accumulated minutes of sedentary behaviour; light, moderate, vigorous and hard activity; and MVPA. The percentage of time spent sedentary, in light intensity activity and in MVPA were calculated using wear time data (the percentage of time spent in hard intensity activity was $<1 \%$, and therefore not reported). The proportion of children attaining different physical activity targets was examined in line with CHMS analyses. For example, Canadian and World Health Organization (WHO) physical activity guidelines recommend 60 minutes of MVPA each day. ${ }^{2,15}$ Like the CHMS analyses, adherence was defined as the probability of accumulating at least 60 minutes of MVPA at least 6 days a week. The probability of accumulating at least 30, 60 and 90 minutes of MVPA on at least $1,2,3,4,5$ or 6 days of the week was also calculated. The probability of accumulating any VPA (at least 5, 10 and 20 minutes) on at least 1, 2, 3, 4, 5 or 6 days of the week was also calculated. Minimal activity was assumed on missing days.

\section{Statistical analyses}

All analyses were conducted using SPSS version 19.0 for Windows (IBM, Armonk, NY, US) and were based on data for participants with at least 4 valid days. Similar to CHMS output, comparisons of physical activity intensity and duration were made according to gender and body weight classification (normal weight, overweight and obese) ${ }^{9}$ using mixedmodel ANOVAs with pair-wise contrasts. Differences between estimates were tested for statistical significance $(p<.05)$.

\section{Results}

\section{Participants}

Table 1 shows a comparison of accelerometer wear by age group and sex between the studies. Table 2 shows the demographic characteristics (gender distribution, mean age, height, weight and BMI) for Project BEAT and CHMS participants.
Hours spent sedentary or in light intensity activity

Project BEAT collected an average of 16.7 hours per day of valid accelerometer data. Children spent an average of 13.3 hours (or $79.6 \%$ of that period) sedentary (790 minutes for boys, 802 minutes for girls; Table 3), a percentage nearly $20 \%$ higher than that identified by Colley et al. ${ }^{4}$ using 2007 to 2009 CHMS data (62\%). Similar to the CHMS, time spent sedentary did not differ by gender or weight classification. While the CHMS dataset did demonstrate differences between genders according to weight classification (with normal weight boys significantly less sedentary than normal weight girls, $p<.05)$, there was no such relationship in Project BEAT. Project BEAT participants spent another 2.9 hours of their day (17.4\% of wear time), on average, in light intensity activity (versus 4 hours per Colley et al. ${ }^{4}$ ); only in Project BEAT did gender differences in the accumulation of light intensity activity appear, with boys accumulating an average of 20 minutes more light intensity activity per day than girls $(p<.05$, Table 3). In both datasets, children classified as either overweight or obese accumulated a similar amount of light intensity activity per day compared to normal weight children.

\section{Moderate-to-vigorous and vigorous activity}

Boys achieved just over half the recommended levels of MVPA per day (35 minutes) while girls attained just 24 minutes per day, findings lower than those reported by Colley et al. ${ }^{4}$ based on the CHMS (average of 61 and 47 minutes, respectively), yet similar with respect to gender differences. As observed in the CHMS, overweight and obese boys in Project BEAT accumulated less MVPA (32 and 26 minutes per day, respectively) compared with boys who were normal weight (38 minutes). Unlike the CHMS, this gradient was also observed in girls; girls classified as being overweight or obese accumulated 4 to 5 minutes less MVPA per day compared with normal weight girls (Table 3).

Project BEAT and CHMS data both revealed that the vast majority of all 
TABLE 3

Average daily minutes of activity (at various levels of intensity) of Project BEAT and CHMS participants, by gender, age and BMI category

\begin{tabular}{|c|c|c|c|c|c|c|}
\hline \multirow[b]{2}{*}{$\begin{array}{l}\text { Study, Sex, Age, } \\
\text { BMI category }\end{array}$} & \multicolumn{6}{|c|}{ Intensity of activity, average minutes/day } \\
\hline & Sedentary & Light & Moderate & Vigorous & Hard & MVPA \\
\hline \multicolumn{7}{|l|}{ Project BEAT } \\
\hline \multicolumn{7}{|l|}{ Boys } \\
\hline Age $10-12$ years & 790 & $185^{\mathrm{a}}$ & $27^{\mathrm{a}}$ & $7^{\mathrm{a}}$ & 1 & $35^{\mathrm{a}}$ \\
\hline \multicolumn{7}{|l|}{ BMI category } \\
\hline Normal weight ${ }^{\mathrm{b}}$ & 786 & $185^{\mathrm{a}}$ & $29^{\mathrm{a}}$ & $8^{\mathrm{a}}$ & 1 & $38^{\mathrm{a}}$ \\
\hline Overweight & 796 & 184 & $25^{c}$ & $6^{c}$ & $<1$ & $32^{c}$ \\
\hline Obese & 800 & 186 & $21^{\mathrm{c}}$ & $4^{c}$ & $<1$ & $26^{c}$ \\
\hline \multicolumn{7}{|l|}{ Girls } \\
\hline Age $10-12$ years & 802 & 165 & 18 & 5 & $<1$ & 24 \\
\hline \multicolumn{7}{|l|}{ BMI category } \\
\hline Normal weight ${ }^{\mathrm{b}}$ & 799 & 165 & 19 & 5 & 1 & 25 \\
\hline Overweight & 808 & 163 & $16^{\mathrm{c}}$ & $4^{c}$ & $<1^{\mathrm{c}}$ & $21^{c}$ \\
\hline Obese & 830 & 174 & 16 & $3^{c}$ & $<1^{\mathrm{C}}$ & $20^{c}$ \\
\hline \multicolumn{7}{|l|}{ CHMS } \\
\hline \multicolumn{7}{|l|}{ Boys } \\
\hline Age 6-10 years ${ }^{b}$ & 445 & 298 & $67^{\mathrm{a}}$ & 2 & - & $69^{\mathrm{a}}$ \\
\hline Age $11-14$ years & $524^{c}$ & $252^{c}$ & $58^{\mathrm{a}}$ & 2 & - & $59^{\mathrm{a}}$ \\
\hline \multicolumn{7}{|l|}{ BMI category ${ }^{\mathrm{d}}$} \\
\hline Normal weight ${ }^{b}$ & $500^{\mathrm{a}}$ & 262 & $64^{\mathrm{a}}$ & 2 & - & $65^{\mathrm{a}}$ \\
\hline Overweight & 524 & 260 & $50^{c}$ & $1^{\mathrm{c}}$ & - & $51^{c}$ \\
\hline Obese & 536 & 248 & $43^{c}$ & $<1^{\mathrm{c}}$ & - & $44^{c}$ \\
\hline \multicolumn{7}{|l|}{ Girls } \\
\hline Age 6-10 years ${ }^{b}$ & 446 & 306 & 56 & 2 & - & 58 \\
\hline Age $11-14$ years & $527^{c}$ & $250^{c}$ & $46^{c}$ & $2^{\mathrm{E}}$ & - & $47^{c}$ \\
\hline \multicolumn{7}{|l|}{ BMI category ${ }^{d}$} \\
\hline Normal weight ${ }^{\mathrm{b}}$ & 524 & 249 & 46 & 2 & - & 48 \\
\hline Overweight & 515 & 262 & 43 & $1^{\mathrm{E}}$ & - & 44 \\
\hline Obese & 544 & 263 & 47 & $<3$ & - & 48 \\
\hline
\end{tabular}

Sources: Built Environment and Active Transport (BEAT) Project (2010-2011); 2007-2009 Canadian Health Measures Survey (CHMS). ${ }^{4}$

Abbreviations: BEAT, Built Environment and Active Transport; BMI, body mass index; CHMS, Canadian Health Measures Survey; MVPA, moderate-to-vigorous physical activity.

a Significantly different from estimate for girls $(p<.05)$.

b Reference category; International Obesity Task Force classification. ${ }^{9}$

c Significantly different from estimate for reference category $(p<.05)$.

d Includes additional age group (aged 15-19 years) sampled in CHMS. Remainder of table reflects results for those aged 6-10 years and 11-14 years, in light of Project BEAT's sample demographics (age 10-12 years).

E Use with caution.

MVPA is accumulated at moderate intensity ( $80 \%$ and $97 \%$, respectively). Around $4.3 \%$ of children in Project BEAT accumulated at least 20 minutes of VPA at least 3 days a week, a result quite similar to CHMS findings (4\%) (Figure 1$)$. $^{*}$
BEAT children $(27.1 \%)$ accumulated at least 10 minutes of VPA on at least 3 days of the week $(35.1 \%$ of boys and $20.4 \%$ of girls; $p<.05)$. Nearly two-thirds of children (64.7\%) accumulated at least 5 minutes of VPA on at least 3 days of the week $(72.8 \%$ of boys and $57.9 \%$ of girls; $p<.05)$, findings that are proportionately higher compared to CHMS observations (Figure 1).

While the vast majority of children in both datasets did not meet the current physical activity recommendations of at least 60 minutes of MVPA at least 6 days a week, the proportion achieving recommendations was lower in Project BEAT ( $<1 \% ; 0.5 \%$ of boys, no girls) in comparison to the CHMS $(6.7 \%$ of children; $9.0 \%$ of boys, $4.1 \%$ of girls) (Table 4). Like the CHMS, the difference in the proportion of Project BEAT children who met guidelines on at least 3 days a week compared to at least 6 days a week was much greater for boys than girls (13.3\% and $2.1 \%$ increase, respectively; Figure 2).

In both datasets, considerably higher percentages of children accumulated 30 minutes of MVPA per day; in Project BEAT, $22.6 \%$ of boys and $5.4 \%$ of girls do so at least 6 days a week (CHMS: $29.0 \%$ and $21.3 \%$, respectively; Table 4 ). Like the CHMS (82.6\%), the majority of boys in Project BEAT (71.8\%) accumulated 30 minutes of MVPA at least 3 days a week; yet unlike the CHMS $(72.6 \%)$, this was not the case for girls (36.9\%). In fact, just over half of Project BEAT girls $(52.6 \%)$ only managed to accumulate 30 minutes of MVPA on 2 or more days of the week.

Not a single child in Project BEAT (and fewer than $2 \%$ in the CHMS) accumulated at least 90 minutes of MVPA at least 6 days of the week and only $2 \%$ met these criteria on at least 2 days of the week $3.3 \%$ of boys, $0.9 \%$ of girls; $p<.05$, Table 4 ). The proportion of children attaining these standards rose to $16.8 \%$ for at least 1 day of the week, with approximately $10 \%$ more boys than girls doing so $(22.3 \%$ and $12.3 \%$, respectively; $p<.05$ ), an increase

\footnotetext{
* The CHMS results in Figure 1 are based on children and youth aged 6-19 years, whereas Project BEAT results are based on children aged 10-12 years.
} 
FIGURE 1

Percentage of Project BEAT participants (10-12 years) and CHMS participants (6-19 years) with at least 5, 10 and 20 minutes of vigorous physical activity a day, by number of days a week

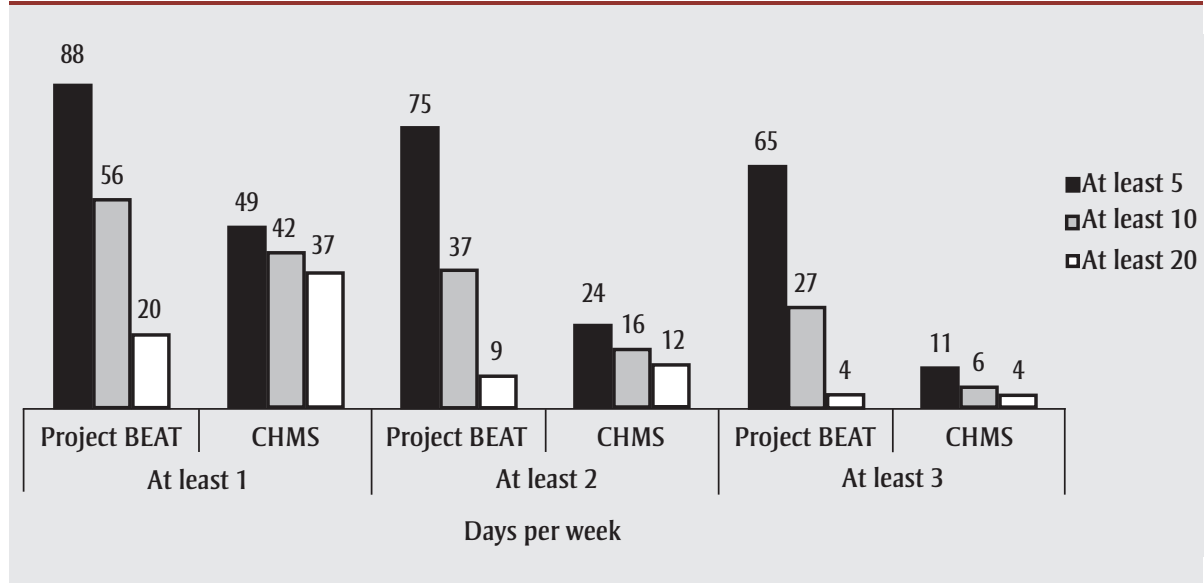

Sources: Built Environment and Active Transport (BEAT) Project (2010-2011); 2007-2009 Canadian Health Measures Survey (CHMS). ${ }^{4}$

FIGURE 2

Percentage of Project BEAT participants (10-12 years) and CHMS participants (6-10 years and 11-14 years) with at least 60 minutes of moderate-to-vigorous physical activity, by days per week and by sex

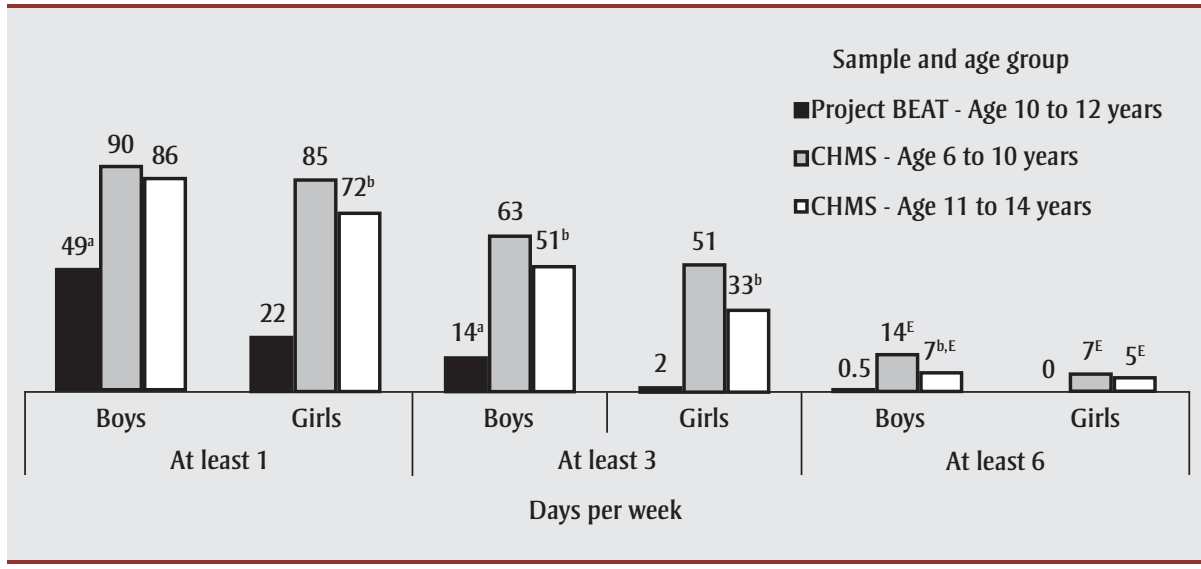

Sources: Built Environment and Active Transport (BEAT) Project (2010-2011); 2007-2009 Canadian Health Measures Survey (CHMS). ${ }^{4}$

${ }^{a}$ Significantly different from estimate for girls $(p<.05)$, Project BEAT.

${ }^{\mathrm{b}}$ Significantly different from estimate for 6 - to 10 -year olds of same sex $(p<.05)$, CHMS.

EUse with caution.

much lower than that reported in the CHMS dataset (60\%; Table 4).

\section{Discussion}

To our knowledge, this is the first attempt to compare accelerometer-measured physical activity data from a large sample with results from a nationally representative dataset (the CHMS, $\mathrm{n}=1608$ ). ${ }^{4}$ Overall, findings were broadly consistent (i.e. similar trends appeared) with some deviations probably due to differences in data collection and reduction protocols and in sample demographics.

Strengths of this study include the large sample size (856 children) and use of an objective measure of physical activity to examine multiple aspects of physical activity behaviour. Our collection of high-frequency physical activity data was particularly appropriate for quantifying children's activity behaviour. ${ }^{13}$ In addition, a relatively low number of participants were excluded due to invalid accelerometer wear (less than 15\%). The biggest limitation of the study is the contextual, sampling and methodological differences between the two datasets, which posed challenges in making direct comparisons. For example, the narrow age range of children sampled and the investigation of Toronto neighbourhoods in Project BEAT was quite different to the national data collection strategy of the CHMS. These issues limit the generalizability of findings to other age groups and geographic locations. While both studies present objectively measured data, the accelerometer measurement and data reduction protocols differed. The lack of standardized physical activity measurement and data reduction protocols in the field are a limitation to any study that attempts to compare results to an independent dataset. Despite these differences, overall trends were similar between the two studies, which supports the notion of very few children $(<10 \%)$ accumulating enough daily activity for health benefits and too many spending a significant amount of their day sedentary.

With methodological differences between Project BEAT and the CHMS acknowledged, the similar trends in findings are sobering. The Canadian physical activity guidelines for children and youth (which are in line with the World Health Organization Global Physical Activity Recommendations ${ }^{15}$ ) encourage children and youth to accumulate at least 60 minutes of MVPA every day. ${ }^{2}$ CHMS data illustrate that only $7 \%$ achieve these recommendations, while Project BEAT data show that even less do so $(<1 \%)$. In fact, not a single girl managed to accumulate at least 60 minutes of MVPA every day of the week, based on Project BEAT data. Perhaps just as sobering is the finding that only $13.2 \%$ of children in Project BEAT managed to attain at least 30 minutes of MVPA on at least 6 days of the week, lower than the CHMS results at $25.3 \%$. BEAT and CHMS data both illustrate that children and youth spend the majority of their day sedentary (anywhere from $62 \%$ to $80 \%$ ). 
TABLE 4

Percentage of Project BEAT participants (10-12 years) and CHMS participants (6-19 years) attaining selected physical activity criteria

\begin{tabular}{|c|c|c|c|c|c|c|c|}
\hline \multirow[b]{2}{*}{ Minutes of MVPA } & \multicolumn{7}{|c|}{ Days active out of $7, \%$} \\
\hline & $\mathbf{0}$ & $\geq 1$ & $\geq 2$ & $\geq 3$ & $\geq 4$ & $\geq 5$ & $\geq 6$ \\
\hline \multicolumn{8}{|l|}{ Project BEAT } \\
\hline \multicolumn{8}{|l|}{$\geq 30$} \\
\hline Total & 22.0 & 78.0 & 65.2 & 52.8 & 40.9 & 25.0 & 13.2 \\
\hline Boys & $11.0^{*}$ & $89.0^{*}$ & $80.3^{*}$ & $71.8^{*}$ & $59.5^{*}$ & $39.2^{*}$ & $22.6^{*}$ \\
\hline Girls & 31.1 & 68.9 & 52.6 & 36.9 & 25.3 & 13.1 & 5.4 \\
\hline \multicolumn{8}{|l|}{$\geq 60$} \\
\hline Total & 66.0 & 34.0 & 15.4 & 7.5 & 3.4 & 1.4 & 0.2 \\
\hline Boys & $51.0^{*}$ & $49.0^{*}$ & $26.2^{*}$ & $13.8^{*}$ & $6.2^{*}$ & $2.8^{*}$ & 0.5 \\
\hline Girls & 78.5 & 21.5 & 6.4 & 2.1 & 1.1 & 0.2 & 0.0 \\
\hline \multicolumn{8}{|l|}{$\geq 90$} \\
\hline Total & 83.2 & 16.8 & 2.0 & 0.4 & 0.1 & 0.0 & 0.0 \\
\hline Boys & $77.7^{*}$ & $22.3^{*}$ & $3.3^{*}$ & 0.5 & 0.3 & 0.0 & 0.0 \\
\hline Girls & 87.7 & 12.3 & 0.9 & 0.2 & 0.0 & 0.0 & 0.0 \\
\hline \multicolumn{8}{|l|}{$\begin{array}{l}\text { CHMS } \\
\geq 30\end{array}$} \\
\hline Total & 5.1 & 94.9 & 87.6 & 77.7 & 64.5 & 47.1 & 25.3 \\
\hline Boys & 3.3 & $96.7^{*}$ & $91.1^{*}$ & $82.6^{*}$ & $70.1^{*}$ & $52.6^{*}$ & $29.0^{*}$ \\
\hline Girls & 6.9 & 93.1 & 83.9 & 72.6 & 58.4 & 41.2 & 21.3 \\
\hline \multicolumn{8}{|l|}{$\geq 60$} \\
\hline Total & 20.2 & 79.8 & 61.3 & 44.4 & 29.3 & 16.6 & 6.7 \\
\hline Boys & 14.8 & $85.2^{*}$ & $69.5^{*}$ & $52.9^{*}$ & $36.4^{*}$ & $21.5^{*}$ & $9.0^{*}$ \\
\hline Girls & 26.1 & 73.9 & 52.6 & 35.4 & 21.7 & 11.3 & $4.1^{\mathrm{E}}$ \\
\hline \multicolumn{8}{|l|}{$\geq 90$} \\
\hline Total & 40.9 & 59.8 & 35.1 & 20.1 & 10.7 & $5.0^{\mathrm{E}}$ & $1.7^{\mathrm{E}}$ \\
\hline Boys & 33.7 & $66.3^{*}$ & $42.5^{*}$ & $26.0^{*}$ & $14.7^{*}$ & $7.1^{* \mathrm{E}}$ & $2.5^{* \mathrm{E}}$ \\
\hline Girls & 47.1 & 52.9 & 27.3 & 13.7 & $6.5^{\mathrm{E}}$ & $2.7^{\mathrm{E}}$ & $<2$ \\
\hline
\end{tabular}

Sources: Built Environment and Active Transport (BEAT) Project (2010-2011); 2007-2009 Canadian Health Measures Survey (CHMS). ${ }^{4}$

Abbreviations: BEAT, Built Environment and Active Transport; CHMS, Canadian Health Measures Survey; MVPA, moderateto-vigorous physical activity.

* Significantly different from estimate for girls $(p<.05)$.

E Use with caution.

\section{Comparing CHMS and BEAT accelerometry procedures}

Proportions attaining physical activity recommendations differed in both studies. Three methodological differences highlight the lack of standardization in accelerometrybased physical activity measurement protocols, which continues to make comparability between studies difficult.

1. The accelerometer wear protocols differed In Project BEAT, participants were asked to wear their accelerometer while awake and asleep to maximize compliance and thus boost the probability of generating a large sample of participants with valid data for inclusion in data analyses. The CHMS requested that participants wear their accelerometer during waking hours only. In Project BEAT, the decision was made to exclude periods of 30 consecutive minutes of zero counts (most of which occurred during sleep time), whereas the CHMS used a less conservative approach. These decisions affect wear time and thus explain the difference in average wear times (Project BEAT: 16.7 hours; CHMS: 13.6 hours). These discrepancies also provide some rationale for differences seen in the proportion of the day spent sedentary and in light intensity activity and MVPA between the two datasets. For example, Project BEAT data show that children spend $79.6 \%$ of their day sedentary. Another $17.4 \%$ is spent in light intensity activity, with MVPA only contributing to $3 \%$ of the daily profile. In the CHMS dataset, children spent an average of $62 \%$ of their waking hours sedentary, with another $29.4 \%$ and $8.6 \%$ spent in light intensity activity and MVPA, respectively.

\section{The accelerometer devices and activity} intensity classification thresholds differed Project BEAT used ActiGraph GT1M accelerometers to monitor physical activity behaviour, while the CHMS used Actical accelerometers. While the GT1M model is one of the most validated and widely used devices of their kind, a possible limitation is that it measures acceleration in the vertical plane only; the Actical device is omni-directional, allowing it to capture a wider range of movement than a uni-axial device and capture non-ambulatory activities. Despite the theoretical advantage of the Actical accelerometer, in reality both accelerometers provide similar information given that the majority of movement is detected in the vertical plane. ${ }^{16}$ Each accelerometer model provides a unique dimensionless activity count over a user-defined interval (i.e. between 1 and 60 seconds). These raw data are converted to useable information using calibration research that generates model-specific activity intensity thresholds. Consequently, time spent sedentary and in light, moderate, vigorous and hard intensity activity can be computed. The CHMS activity intensity thresholds for the Actical were derived from calibration work in children ${ }^{17,18}$ and adults, ${ }^{18}$ and the Project BEAT thresholds only from calibration trials with children. $^{14}$

Metabolic energy turnover (MET) values are often used to express the intensity of physical activity according to intensity categories; a compendium of energy costs for a variety of children's activities is available. ${ }^{19}$ In most studies like the CHMS, moderate intensity is defined as 3 METs or more. However, more recent evidence suggests that a threshold of 4 METs or 
more may be more appropriate for describing moderate or higher intensity activity in children ${ }^{20-23}$ and for determining relationships between activity and health outcomes. ${ }^{14}$ Indeed, the National Health and Nutrition Examination Survey (NHANES) in the U.S. uses a moderate intensity threshold based on 4 METs to classify MVPA in children ${ }^{24}$; in the BEAT study, the threshold for moderate intensity was also based on 4 METs. Our use of a more stringent threshold to classify MVPA (a decision made before the release of the CHMS findings) likely explains the lower levels of MVPA observed (and fewer children meeting guidelines) in Project BEAT data compared with CHMS data.

Reports in other countries, for example, NHANES $^{7}$ in the U.S. and the Avon Longitudinal Study of Parents and Children (ALSPAC) ${ }^{25}$ in England, support these results. It is of particular interest to compare Project BEAT data with the ALSPAC data since both studies used the same thresholds to classify moderate and vigorous intensity activity: we see very similar proportions of children achieving the 60 minutes of MVPA per day guidelines (BEAT at $<1 \%$ and ALSPAC at $2.5 \%$ ) and similar average levels of MVPA (BEAT at 29 minutes per day and ALSPAC at 20 minutes per day).

\section{The user-specified data collection interval differed}

Project BEAT used a 5-second epoch to capture the short and sporadic bursts of activity that are typical in children, ${ }^{13}$ whereas the CHMS captured physical activity data at 1-minute intervals. The influence of epoch length on physical activity data has been discussed at length: shorter epochs capture more MVPA, and longer epochs "dilute" the intensity of the data $^{26,27}$ and therefore affect the proportion of children attaining PA guidelines. ${ }^{28}$ Some have found significant epoch effects for hard and very hard activity, ${ }^{26}$ and others for all intensities. ${ }^{29}$ Using direct observation, McClain at al. ${ }^{30}$ showed that a 5-second epoch provided the least discrepant estimates of MVPA in fifth grade children compared with 10-, 15-, 20-, 30- and 60-second epochs. Indeed, there is strong support for utilizing a 5-second epoch to truly capture children's spontaneous, discontinuous patterns of activity. ${ }^{13,29,30}$

Project BEAT's finding of a greater proportion of children accumulating at least 5, 10 and 20 minutes of vigorous intensity activity on one or more days in comparison to the CHMS results could be a reflection of utilizing a shorter epoch to capture and express accelerometer data. The discrepancies were more apparent for lower levels of VPA (at least 5 and 10 minutes); in fact, when examining those accumulating at least 20 minutes of VPA per day, the proportions were nearly identical between datasets (around $4 \%$ for each). The epoch effect may be diluted at the upper extremes of daily VPA accumulation and have a less significant impact on levels of MVPA than accelerometer intensity thresholds, given levels were somewhat lower in Project BEAT compared with the CHMS dataset.

\section{Conclusion}

Using data from Project BEAT, this study demonstrates that the low levels of physical activity and high levels of sedentary behaviour amongst Canadian youth, as reported in the CHMS, do occur in an independent sample of Canadian youth. Accelerometry data in both datasets show that the majority of children and youth do not meet current physical activity recommendations and spend a significant proportion of their day sedentary. These similarities have been established despite contextual, sampling and methodological differences between the two datasets, limitations that have been noted and discussed, and also presented as three methodological considerations in analyzing accelerometry data. That both datasets reveal similar trends in physical activity and inactivity behaviour among Canadian children and youth is encouraging for purposes of validation, yet disheartening given the ramifications of such inactivity on health. The consistency of data from the CHMS and that on a sample of children from the Greater Toronto Area, where conditions might be most conducive for physical activity in terms of facilities and resources, supports the notion that physical inactivity is most likely an ongoing problem across Canada.

\section{Acknowledgements}

This research was funded by the Built Environment, Obesity and Health Strategic Initiative of the Heart and Stroke Foundation and the Canadian Institutes of Health Research (CIHR).

\section{References}

1. Janssen I, LeBlanc AG. Systematic review of the health benefits of physical activity and fitness in school-aged children and youth. Int J Behav Nutr Phys Act. 2010;7:40.

2. Canadian Society for Exercise Physiology, ParticipACTION. Canadian Society for Exercise Physiology and ParticipACTION share new research to inform Canadians of physical activity levels required. Press release. 2010 May 12. Available at: http://files.participaction.com/pressreleases /en/csep_pac-nationalrelease-final-en.pdf

3. Katzmarkyk PT, Ardern CI. Physical activity levels of Canadian children and youth: current issues and recommendations. Can J Diabetes. 2004;28:67-78.

4. Colley RC, Garriguet D, Janssen I, Craig CL, Clarke J, Tremblay MS. Physical activity of Canadian children and youth: accelerometer results from the 2007 to 2009 Canadian Health Measures Survey. Health Reports (Statistics Canada, 82-003) 2011; 22:1-10.

5. Colley R, Connor Gorber S, Tremblay MS. Quality control and data reduction procedures for accelerometry-derived measures of physical activity. Health Rep. 2010; 21:63-9.

6. Tremblay MS, Shields M, Laviolette M, Craig CL, Janssen I, Gorber SC. Fitness of Canadian children and youth: results from the 2007-2009 Canadian Health Measures Survey. Health Rep. 2009;21:7-20.

7. Troiano RP, Berrigan D, Dodd K, Masse LC, Tilert T, McDowell M. Physical activity in the United States measured by accelerometer. Med Sci Sports Exerc. 2008;40:181-8. 
8. Veugelers PJ, Fitzgerald AL. Prevalence of and risk factors for childhood overweight and obesity. CMAJ. 2005;173:607-13.

9. Cole TJ, Bellizzi MC, Flegal KM, Dietz WH. Establishing a standard definition for child overweight and obesity worldwide: international survey. BMJ. 2000;320:1240.

10. De Vries SI, Van Hirtum HW, Bakker I, Hopman-Rock M, Hirasing RA, Van Mechelen W. Validity and reproducibility of motion sensors in youth: a systematic update. Med Sci Sports Exerc. 2009;41: 818-27.

11. Chen KY, Bassett DR, Jr. The technology of accelerometry-based activity monitors: current and future. Med Sci Sports Exerc. 2005;37:S490-500.

12. Welk GJ. Principles of design and analyses for the calibration of accelerometry-based activity monitors. Med Sci Sports Exerc. 2005;37:S501-11.

13. Stone MR, Rowlands AV, Middlebrooke AR, Jawis MN, Eston RG. The pattern of physical activity in relation to health outcomes in boys. Int J Pediatr Obes. 2009; 4:306-15.

14. Stone MR, Rowlands AV, Eston RG. Relationships between accelerometerassessed physical activity and health in children: impact of the activity-intensity classification method. J Sports Sci Med. 2009;8:136-43.

15. World Health Organization. Global recommendations on physical activity for health. Geneva (CH): World Health Organization; 2010.

16. Corder K, Brage S, Ekelund U. Accelerometers and pedometers: methodology and clinical application. Curr Opin Clin Nutr Metab Care. 2007;10:597-603.

17. Puyau MR, Adolph AL, Vohra FA, Zakeri I, Butte NF. Prediction of activity energy expenditure using accelerometers in children. Med Sci Sports Exerc. 2004;36: 1625-31.

18. Wong SL, Colley R, Connor Gorber S, Tremblay M. Actical accelerometer sedentary activity thresholds for adults. J Phys Act Health. 2011;8:587-91.
19. Ridley K, Olds TS. Assigning energy costs to activities in children: a review and synthesis. Med Sci Sports Exerc. 2008;40: 1439-46.

20. Harrell JS, McMurray RG, Baggett CD, Pennell ML, Pearce PF, Bangdiwala SI. Energy costs of physical activities in children and adolescents. Med Sci Sports Exerc. 2005;37:329-36.

21. Mattocks C, Leary S, Ness A, Deere K, Saunders J, Tilling K, et al. Calibration of an accelerometer during free-living activities in children. Int $\mathrm{J}$ Pediatr Obes. 2007;2:218-26.

22. Reilly JJ, Penpraze V, Hislop J, Davies G, Grant S, Paton JY. Objective measurement of physical activity and sedentary behaviour: review with new data. Arch Dis Child. 2008;93:614-9.

23. Treuth MS, Schmitz K, Catellier DJ, McMurray RG, Murray DM, Almeida MJ, et al. Defining accelerometer thresholds for activity intensities in adolescent girls. Med Sci Sports Exerc. 2004;36:1259-66.

24. Trost SG, Pate RR, Sallis JF, Freedson PS, Taylor WC, Dowda M, et al. Age and gender differences in objectively measured physical activity in youth. Med Sci Sports Exerc. 2002;34:350-5.

25. Riddoch CJ, Mattocks C, Deere K, Saunders $\mathrm{J}$, Kirkby J, Tilling $\mathrm{K}$, et al. Objective measurement of levels and patterns of physical activity. Arch Dis Child. 2007;92:963-9.

26. Nilsson A, Ekelund U, Yngve A, Sjoestroem M. Assessing physical activity among children with accelerometers using different time sampling intervals and placements. Pediatr Exerc Sci. 2002;14:87-96.

27. Trost SG, McIver KL, Pate RR. Conducting accelerometer-based assessments in fieldbased research. Med Sci Sports Exerc. 2005;37:S531-43.

28. Ojiambo R, Cuthill R, Budd H, Konstabel K, Casajus JA, Gonzalez-Aguero A, et al. Impact of methodological decisions on accelerometer output variables in young children. Int J Obes (London). 2011;35: S98-103.
29. Edwardson CL, Gorely T. Epoch length and its effect on physical activity intensity. Med Sci Sports Exerc. 2010;42:928-34.

30. McClain JJ, Abraham TL, Brusseau TA Jr., Tudor-Locke C. Epoch length and accelerometer outputs in children: comparison to direct observation. Med Sci Sports Exerc. 2008;40:2080-7. 